\title{
Bartonella henselae Biofilm Detected on Catheter of Patient with Persistent Bartonellosis
}

Melissa Weber-Sanders ${ }^{1}$, Paulo ENF Velho ${ }^{2}$, Gislaine Vieira-Damiani ${ }^{2}$, Marilene Neves da Silva ${ }^{2}$, Vitor B. Pelegati ${ }^{3}$, Carlos Lenz Cesar $^{3}$ and Marna Ericson ${ }^{1}$

1. Department of Dermatology, University of Minnesota Medical School, Minneapolis, MN USA.

2. Department of Medicin and Dermatology, UNICAMP Medical Sciences School, Campinas, Brazil

3. National Institute of Science and Technology on Photonics Applied to Cell Biology (INFABIC), UNICAMP Medical Sciences School, Campinas, Brazil

Bartonella spp. can induce long-lasting bacteremia in mammals (1). Bartonella spp. are causative agents of cat-scratch disease, endocarditis, bacillary angiomatosis, bacillary peliosis, trench fever, neurocognitive dysfunction and regional pain syndrome (2). Chronic Bartonella spp. infection and subsequent manifestations of persistent infection may be the result of the bacteria growing in biofilms. In humans, at least four Bartonella species have been associated with infectious endocarditis, a biofilmassociated infectious disease (3). Biofilms are sessile communities of interacting unicellular organisms that adhere to a surface and to each other and create unique, complex structures, covered with a protective layer of extracellular polymeric substances (EPS). Biofilms and their inherent resistance to antimicrobial agents are often key players in many persistent and chronic bacterial infections $(4,5)$. We acquired skin biopsies from 5 patients with blood PCR-positive B. henselae. 60-100 micron-thick sections were immunostained with antibodies to $B$. henselae. Stained sections were imaged using both single- and multi-photon imaging to capture epifluorescence emission and second harmonic generation signal of non-stained collagen I and II (Fig 1). The crystalline triple-helix of native fibrillar collagen (I, II, III and V) generates a strong second harmonic generation (SHG) signal (6). In patients with longstanding bartonellosis, clusters of immuno-reactive $B$. henselae were found intimately associated with fibrillar dermal collagen (Fig 2). Additionally, we found a Bartonella spp. biofilm on a peripheral intravenous catheter line removed from a patient with persistent Bartonella (Fig 3). Further, B. henselae, cultured 14 or 21 days, on collagen I and II-coated coverslips revealed a $B$. henselae biofilm matrix (Fig 4). Identification and characterization of Bartonella spp. biofilms in vivo and in situ will lead to a better understanding of persistent Bartonellosis and provide direction in treatment modalities.

1) Breitschwerdt, E. B. et al (2010). "Bartonellosis: an emerging infectious disease of zoonotic importance to animals and human beings." J Vet Emerg Crit Care (San Antonio) 20(1): 8-30.

2) Harms, A. and C. Dehio (2012). "Intruders below the radar: molecular pathogenesis of Bartonella spp." Clin Microbiol Rev 25(1): 42-78.

3) Pulliainen, A T. and C. Dehio (2012). "Persistence of Bartonella spp. stealth pathogens: from subclinical infections to vasoproliferative tumor formation." FEMS Microbiol Rev 36(3): 563-599.

4) Dunny, G. et al. (2008). "Multicellular behavior in bacteria: communication, cooperation, competition and cheating." Bioessays 30(4): 296-298.

5) Oliveira, A. and L. Cunha Mde (2010). "Comparison of methods for the detection of biofilm production in coagulase-negative staphylococci." BMC Res Notes 3: 260.

6) Kukulski, et al. (2012). "Precise, correlated fluorescence microscopy and electron tomography of lowicryl sections using fluorescent fiducial markers." Methods Cell Biol 111: 235-257. 


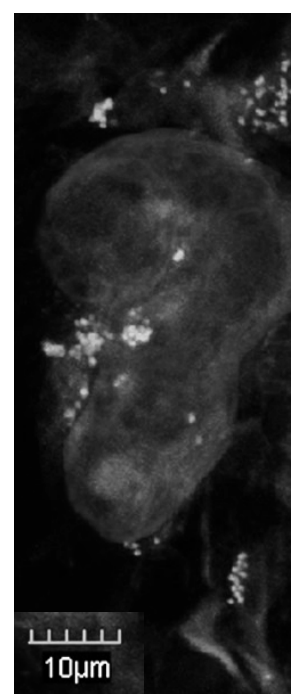

Figure 1: B. henselae immuno-reactivity associated with micro-vasculature in dermal layer of non-lesional skin of patient with PCR blood-positive for $B$. henselae, $B$. vinsonii, and B. spp. Fourmm punch biopsy from nonlesional calf skin was processed, anti-collagen type IV plus anti-goat secondary antibody conjugated to $\mathrm{Cy} 3$ (aqua) and anti-B. henselae made in mouse plus secondary antibody conjugated to Cy5.
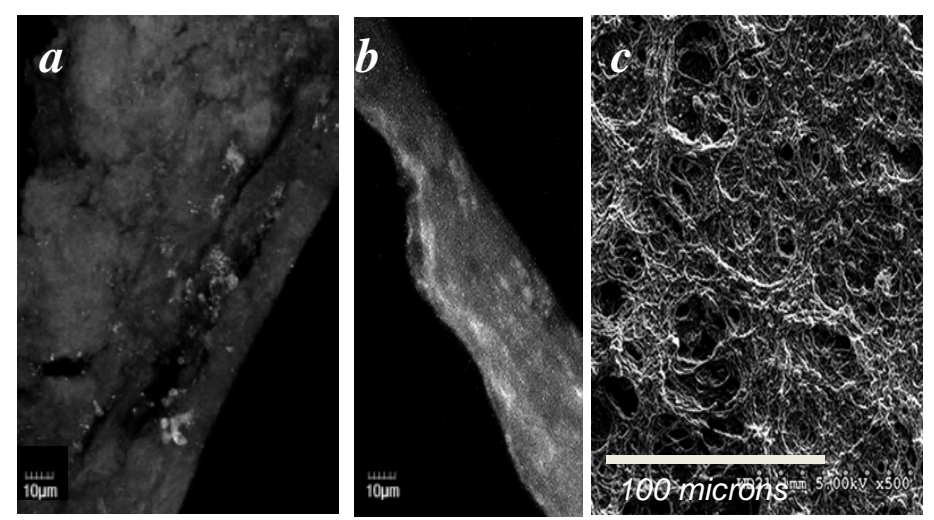

Figure 3. PIC line from patient with persistent Bartonella harbors Bartonella spp. biofilm. a: $B$. henselae-ir of pic line. b: Film-Tracer Sypro-Ruby stains PIC biofilm, c: SEM of same PIC line reveals meshwork of biofilm.

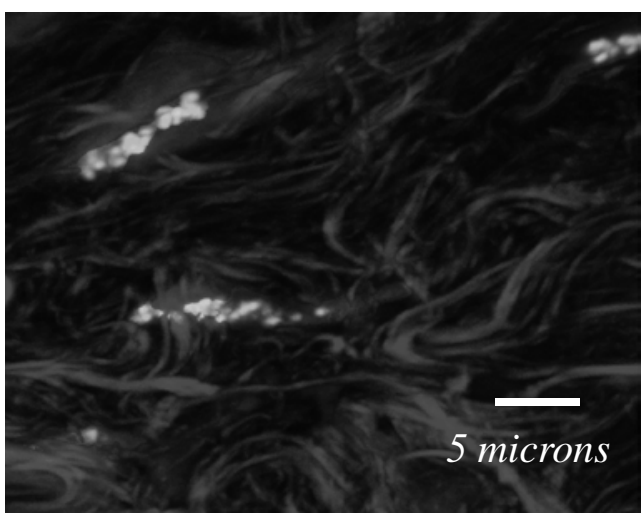

Figure 2: B.henselae intimately associated with collagen fibrils of dermis in non-lesional skin of patient with PCR blood-positive for B. henselae, B. vinsonii, and B. spp. Four-mm punch biopsy from non-lesional calf skin was processed, labeled with anti-B. henselae made in mouse plus secondary antibody conjugated to Cy5. Imaged with two-photon laser scanning microscopy, 60X oil objective and 5Z electronic zoom. Second harmonic generation signal produces robust image of non-stained collagen type I and II (magenta) and two-photon epifluorescent signal of Cy5 is also captured. (Zeiss multi-photon scope images courtesy of Lens Cezar, PhD, UNICAMP, Campinas, Brazil.)

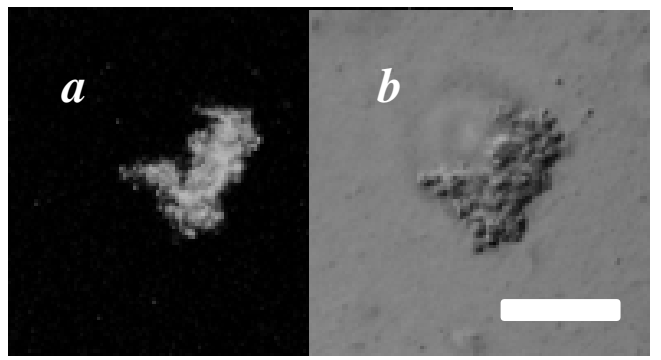

Figure 4: B. henselae immunoreactivity of bacteria stain in B. henselae cultured cells. B. henselae was cultured for $7 \mathrm{D}$, lightly fixed, and stained with combinations of anti- $B$. henselae made in mouse and anti-mouse conjugated to Cy5. All samples imaged using confocal microscopy with PlanApo 60x (oil, NA 1.42). Scale bars = 10 microns. a) Immunoreactive B. henselae made in mouse, anti-mouse conjugated to Cy5 and b) differential interference contrast . 